\title{
Evaluación de las potencialidades de la iniciación al tenis en Brasil
}

\section{Caio Corrêa Cortela, Guy Ginciene, Michel Milistetd, Silvio Pinheiro de Souza y Carlos Adelar Abaide Balbinotti}

\section{RESUMEN}

La iniciación al tenis es una etapa muy importante para el desarrollo de los niños y jóvenes. Las acciones en este periodo pueden determinar si un niño sigue practicando el deporte o no. Un trabajo bien hecho puede, además de formar tenistas de alto rendimiento, determinar la continuidad de jóvenes principiantes en el tenis para toda la vida.

\author{
Palabras clave: tenis; \\ iniciación deportiva; \\ Tennis10s. \\ Recibido: 3 Diciembre 2018 \\ Aceptado: 8 Enero 2019 \\ Autor correspondiente: \\ Caio Corrêa Cortela. \\ Correo electrónico: \\ capacitacao@fpt.com.br
}

\section{INTRODUCCIÓN}

Hace poco más de 10 años la ITF lanzó la campaña "Play and Stay". El objetivo principal de esta acción era promover el tenis en todo el mundo, aumentando la participación y la tasa de retención. Dentro de la propia campaña, el trabajo con niños de hasta 10 años de edad - que más tarde pasó a ser conocido como Tennis10s - se convirtió en el elemento más conocido. La propuesta de las etapas rojas, naranjas y verdes, además de las adaptaciones de materiales para grupos de edad diferentes, se divulgaron ampliamente, y mensajes claves como: "el tenis es divertido"; "el tenis es fácil"; "el tenis competitivo es fácil"; "el tenis es sano"; y "el tenis es un deporte para todos"; empezaron a llegar a los entrenadores (Buszard et al., 2018).

Ante la evidencia de que una experiencia positiva en la iniciación a un nuevo deporte puede influir de forma positiva la participación deportiva, o incluso el desarrollo personal mediante el desarrollo de habilidades para la vida, parece fundamental conocer la realidad de la situación en nuestro propio país, en este caso Brasil, con el fin de motivar propuestas futuras, investigación, formación de tutores y otras intervenciones en el campo del entrenamiento. En este sentido, este artículo tiene el objetivo de describir las potencialidades de la estructura brasileña de la iniciación al tenis, como señalaron los profesionales responsables de realizar los programas introductorios dirigidos a los niños.

\section{MÉTODOS}

Esta investigación, de carácter cualitativo (Sparkes \& Smith, 2014), busca estudiar las percepciones de los coordinadores responsables de los programas introductorios de tenis. Para ello, se entrevistaron 14 coordinadores de clubes tradicionalmente reconocidos en el campo de iniciación deportiva y formación de jugadores, nombrados por la Confederación Brasileña de Tenis (CBT).

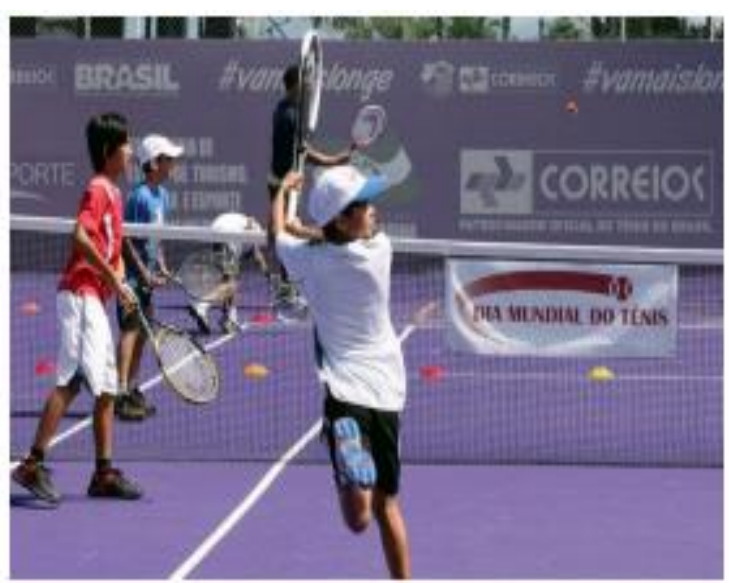




\begin{tabular}{|c|c|}
\hline \multicolumn{2}{|c|}{ Potencialidades: Iniclación al tenis en Brasill } \\
\hline Coordinadores & Categorias \\
\hline$(s 1 ; s 2 ; s 3 ; s 6 ; s 11 ; \$ 12)$ & $\begin{array}{l}\text { Usodemateriales adaptados yel programa } \\
\text { Tennis } 10 \mathrm{~s}\end{array}$ \\
\hline$(\$ 1 ; 54 ; 55 ; \$ 6)$ & Formación de los entrenadores nacionales \\
\hline$(\$ 1 ; 52 ; 59)$ & Mayor acceso a la informadón \\
\hline
\end{tabular}

Figura 1 Categorias de andilisis.

Para analizar los resultados, se utilizó el "análisis temático" (Sparkes \& Smith, 2014) que agrupó las respuestas de las entrevistas en temas similares para posibilitar la presentación y discusión de los resultados. Los puntos citados por al menos tres coordinadores fueron incluidos y considerados para análisis. En este sentido, tres categorías emergieron del análisis, como puede verse en la figura 1.

\section{RESULTADOS Y DISCUSÍON}

La utilización de materiales adaptados e ideas centrales del programa Tennis10s aparecen como puntos de mayor destaque en las respuestas de los coordinadores:

[...] lo que creo que fue el gran cambio en estos últimos años fue la utilización del material adaptado. Es algo que vino poco a poco, con mucha resistencia en clubes tradicionales como el nuestro. (S12 - 13'20")

[...] creo que hemos avanzado mucho, [por ejemplo] con el uso de las etapas: la etapa Roja, la etapa Naranja, la bola Verde, y luego el paso a la cancha grande. Mucha gente utiliza esto. Eso es bueno, es un punto positivo. ( $\left.\mathrm{S} 11-12^{\prime} 30^{\prime \prime}\right)$.

A pesar del número limitado de estudios que investigan el impacto del uso de materiales adaptados en la adquisición/ desarrollo de fundamentos técnicos y tácticos relacionados con el deporte, los resultados encontrados hasta el momento evidencian los efectos positivos de esa práctica, favoreciendo un aprendizaje más rápido y la adopción de patrones técnicos y tácticos más avanzados y representativos del juego a ser jugado en el futuro (Buszard et al., 2016).

Además de la utilización de materiales adaptados, los coordinadores también mencionaron otros elementos del programa Tennis 10 s, como el uso de progresiones entre etapas y de adaptaciones metodológicas, tales como la enseñanza enfocada en el juego, la comprensión de los aspectos tácticos y la contextualización de la enseñanza técnica. El hecho de que las ideas centrales del programa de Tennis10s estén presentes en el discurso de los coordinadores es un punto positivo que señala hacia la adopción de criterios mínimos que caracterizan la iniciación deportiva en Brasil, así como el establecimiento de directrices nacionales que pueden fundamentar las primeras etapas del programa.

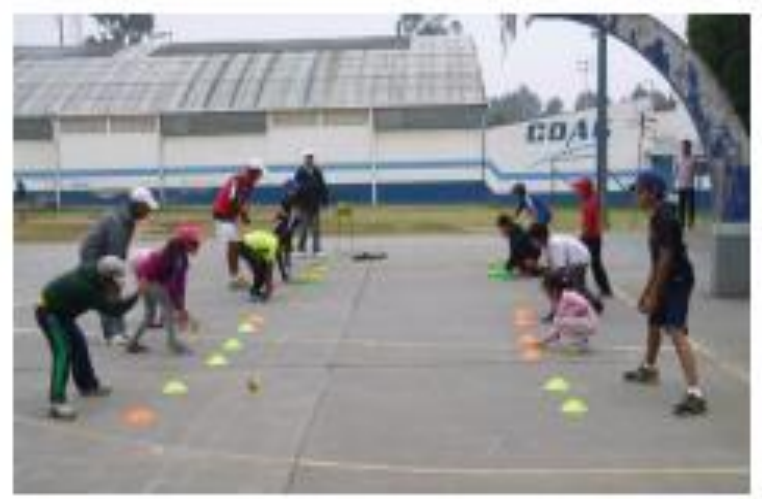

la iniciación deportiva en Brasil, así como el establecimiento de directrices nacionales que pueden fundamentar las primeras etapas del programa.

Los principios pedagógicos presentes en el Tennis10s se encuentran alineados a las propuestas contemporáneas de la enseñanza de los juegos deportivos, como el 'Teaching Games for Understanding' (TGfU) (Cortela et al., 2012; Thorpe, Bunker \& Almond, 1986), por ejemplo, y sus variaciones (Harvey \& Jarrett, 2013). Estas propuestas actúan como un modelo genérico capaz de servir como el eje central para la "estandarización" de la enseñanza del deporte en el país.

El segundo punto clave destacado en las respuestas de los coordinadores se refiere a la formación de los entrenadores involucrados en el proceso:

[...] creo que la formación de los profesionales que están trabajando en este ámbito es mucho más desarrollada ahora de lo que fue hace cinco o diez años. Entonces este elemento de la formación profesional es un punto muy positivo porque hoy en día tiene un entrenador que se encarga de ese ámbito [de iniciación al tenis], que se encarga del programa de la iniciación al tenis de un club. Él/ella está centrado/a en la iniciación y es muy competente. Eso no teníamos en el pasado. (S1 - 18'30").

De hecho, las oportunidades de aprendizaje en el contexto formal y no formal han ampliado en las últimas dos décadas. En el contexto formal, el requisito de tener una formación inicial en Educación Física para actuar como un entrenador de tenis en Brasil (un requisito desde 1998) ha impactado el número de profesionales graduados, causando un cambio en el perfil de los entrenadores que trabajan en el ámbito (Cortela et al., 2013) y un cambio en la autopercepción de competencia relacionada a los conocimientos y habilidades requeridos para la actuación profesional (Cortela et al., 2017).

En el contexto no formal, las oportunidades de aprendizaje se encuentran asociadas con las operaciones del Departamento de Capacitación de Entrenadores de la Confederación Brasileña de Tenis (CBT). Actualmente, el programa ofrecido por la CBT involucra más de 300 horas de formación, abarcando desde el trabajo de iniciación hasta el alto rendimiento, siendo la 
actividad de formación continuada más frecuentada por los entrenadores brasileños (Cortela et al., 2013).

El tercer punto considerado por los coordinadores fue la mayor facilidad en el acceso a la información. Según los coordinadores, el acceso a los estudios, y principalmente a Internet, ha ampliado la gama de información disponible y reducido el tiempo que tarda ponerles al día con los profesionales en el trabajo:

[...] el otro punto clave es que el Internet, y principalmente YouTube, posibilita que todo el mundo vea lo que está siendo hecho en otros lugares. Y se puede, dependiendo en su interés, poner esas cosas en práctica. Creo que este es un punto clave. (S2 - 8'04").

La utilización del Internet para buscar materiales de apoyo es una de las fuentes principales del aprendizaje informal usadas por los entrenadores. Al buscar información en la red, los entrenadores a menudo buscan soluciones a problemas cotidianos que enfrentan en la práctica profesional, algo que le da mayor significado al aprendizaje (Walker et al., 2018).

En el caso específico de los entrenadores brasileños, el internet no solo ha reducido la brecha con respecto al tiempo, sino también el gap de accesibilidad ya que es menos costoso acceder la información. Las plataformas como Tennis iCoach permiten a los entrenadores seguir, casi simultáneamente, los principales eventos de formación y las tendencias observadas en otros países.

\section{CONCLUSÍON}

Las percepciones de los coordinadores apuntan a algunos puntos claves sobre la iniciación deportiva brasileña con respecto al tenis. Además, se observa que algunas acciones realizadas en años recientes, como el fomento del programa Tennis10s y los cursos de formación, parecen estar contribuyendo directamente a la práctica pedagógica de los entrenadores. Esta información es relevante para evaluar las acciones tomadas hasta el momento y aún pueden contribuir a las futuras intervenciones.

Nota: los autores agradecen el apoyo financiero y logístico de la Confederación Brasileña de Tenis para el desarrollo de este estudio.

\section{REFERENCIAS}

Buszard, T., Farrrow, D., \& Westerbeek, H. (2018). Evaluation of the ITF Tennis Play and Stay campaing since its inception in 2007. Nieuwegein: Arko Sports Media.

Buszard, T.; Reid, M.; Masters, R.; Farrow, D. (2016). Scaling the Equipment and Play Area in Children's Sport to improve Motor Skill Acquisition: A Systematic Review. Sports Med. 46(6), 829-843. https://doi.org/10.1007/s40279-015-0452-2
Cortela, C. C., Fuentes, J. P., Aburachid, L. M. C., Kist, C., \& Cortela, D. N. R. (2012). Sport initiation to tennis: an example of the Play and Stay program in the light of the pedagogy of the sport Conexões, 10(2), 214-234. https://doi.org/10.20396/conex.v10i2.8637683

Cortela, C. C., Aburachid, L. M. C., Sousa, S. P., Cortela, D. N. R., \& Fuentes, J. P. (2013). Initial and continuing training of tennis coaches in Parana State. Conexões, 11(2), 60-84. https://doi.org/10.20396/conex.v11i2.8637617

Cortela, C. C., Balbinotti, C. A. A. B., Tozetto, V. B., Both, J., Milistetd, M. (2017). Association between Initial Training and Self-Perception of Professional Competence in Tennis Coaches. Journal of Sport Pedagogy and Research, 3(2), 32- 42.

Coté, J. Turnnidge, J. Evans, M. B. (2014). The dynamic process of development through sport. Kinesiologia Slovenica, 20(3), 14-26.

Harvey, S., \& Jarrett, K. (2013). A review of the game-centred approaches to teaching and coaching literature since 2006. Physical Education \& Sport Pedagogy, 1-23. https://doi.org/10.1080/17408989.2012.754005

Sparkes, A. C., \& Smith, B. (2014). Qualitative research methods in Sport, Exercise and Health. London: Routledge. https://doi.org/10.4324/9780203852187

Thorpe, R., Bunker, D., \& Almond, L. (1986). Rethinking games teaching. Leicstershire: Univesity of Loughborough.

Walker. L. F; Thomas, R.; Driska, A. P. (2018). Informal and nonformal learning for sport coaches: A systematic review. International Journal of Sports Science \& Coaching. https://doi.org/10.1177/1747954118791522

CONTENIDO ITF ACADEMY RECOMENDADO (HAZ CLICK ABAJO)

\section{TTF Academy}

Derechos de Autor (c) 2019 Caio Corrêa Cortela, Guy Ginciene, Michel Milistetd,

Silvio Pinheiro de Souza y Carlos Adelar Abaide Balbinotti

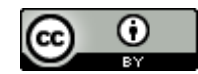

Este texto está protegido por una licencia CreativeCommons 4.0.

\footnotetext{
Usted es libre para Compartir —copiar y redistribuir el material en cualquier medio o formato- y Adaptar el documento - remezclar, transformar y crear a partir del material- para cualquier propósito, incluso para fines comerciales, siempre que cumpla la condición de:

Atribución: Usted debe dar crédito a la obra original de manera adecuada, proporcionar un enlace a la licencia, e indicar si se han realizado cambios. Puede hacerlo en cualquier forma razonable, pero no de forma tal que sugiera que tiene el apoyo del licenciante o lo recibe por el uso que hace de la obra. Resumendelicencia - Textocompletodelalicencia
} 